\title{
Validity of SyMRI for Assessment of the Neonatal Brain
}

\author{
Victor Schmidbauer ${ }^{1}$ Gudrun Geisl' • Mariana Cardoso Diogo ${ }^{1}$ Suren Jengojan ${ }^{1}$. Vsevolod Perepelov ${ }^{1}$. \\ Michael Weber $^{1} \cdot$ Katharina Goeral $^{2}$. Florian Lindenlaub ${ }^{1} \cdot$ Katrin Klebermass-Schrehof $^{2} \cdot$ Angelika Berger $^{2}$. \\ Daniela Prayer ${ }^{1} \cdot$ Gregor Kasprian $^{1}$
}

Received: 28 October 2019 / Accepted: 24 February 2020 / Published online: 11 March 2020

(c) The Author(s) 2020

\begin{abstract}
Purpose The purpose of this study was to assess the diagnostic accuracy of T1-weighted and T2-weighted contrasts generated by the MR data postprocessing software SyMRI (Synthetic MR AB, Linköping, Sweden) for neonatal brain imaging.

Methods In this study 36 cases of neonatal MRI were retrospectively collected, which included T1-weighted and T2-weighted sequences as well as multi-dynamic multi-echo (MDME) sequences. Of the 36 neonates 32 were included in this study and 4 neuroradiologists independently assessed neonatal brain examinations on the basis of conventional and SyMRI-generated T1-weighted and T2-weighted contrasts, in order to determine the presence or absence of lesions. The sensitivity and specificity of both methods were calculated and compared.

Results Compared to conventionally acquired T1 and T2-weighted images, SyMRI-generated contrasts showed a lower sensitivity but a higher specificity (SyMRI sensitivity 0.88, confidence interval (CI): 0.72-0.95; specificity 1, CI: 0.89-1/conventional MRI: sensitivity: 0.94, CI: 0.80-0.98; specificity: 0.94, CI: 0.80-0.98).

Conclusion The T1-weighted and T2-weighted images generated by SyMRI showed a diagnostic accuracy comparable to that of conventionally acquired contrasts. In addition to semiquantitative imaging data, SyMRI provides diagnostic images and leads to a more efficient use of available imaging time in neonatal brain MRI.
\end{abstract}

Keywords Magnetic resonance imaging $\cdot$ Newborn $\cdot$ Software $\cdot$ Sensitivity $\cdot$ Specificity

\section{Abbreviations}

B1 Local radiofrequency field

CI Confidence interval

$\mathrm{C \kappa} \quad$ Cohen coefficient

DWI Diffusion-weighted imaging

FK Fleiss coefficient

FLAIR Fluid-attenuated inversion recovery

MDME Multi-dynamic multi-echo

MRI Magnetic resonance imaging

PD Proton density

SE Spin echo

Gregor Kasprian

gregor.kasprian@meduniwien.ac.at

1 Department of Biomedical Imaging and Image-guided Therapy, Medical University of Vienna, Währinger Gürtel 18-20, 1090 Vienna, Austria

2 Department of Pediatrics and Adolescent Medicine, Comprehensive Center for Pediatrics, Medical University of Vienna, Währinger Gürtel 18-20, 1090 Vienna, Austria
SWI Susceptibility-weighted imaging

TE Echo time

TR Repetition time

TSE Turbo spin echo

\section{Introduction}

Brain imaging is essential in neonatal neuropediatric diagnostics [1]. In neonates, magnetic resonance imaging (MRI) serves as a reliable modality for the detection of brain pathologies, as well as for the assessment of myelination [2-6]. Based on conventional MRI findings, substantial information about the future course of neurodevelopment can be obtained [2, 7-9]. Apart from deficient myelination, brain injury, e.g., due to hemorrhage, asphyxia, or infarction during the prenatal and neonatal period, may severely impair brain maturation $[6,8,10-12]$. Therefore, a precise visualization of pathological changes must be the aim of imaging methods, in order to ensure an optimal individual diagnosis and follow-up. 
Conventional MRI is a meaningful, yet highly time-consuming method, for brain imaging. Primarily in a pediatric cohort, a long examination time may overtax patient compliance. Hence, it is of paramount importance to develop imaging techniques that enable a reduction of acquisition time.

Based on a single, quantitative, multi-dynamic multiecho (MDME) sequence, the MR data post-processing software SyMRI (Synthetic MR AB, Linköping, Sweden) enables the generation of various MR contrasts, such as T1weighted, T2-weighted, proton density (PD)-weighted, and inversion recovery [13], which bears the potential to reduce examination time [14-16]. Moreover, SyMRI allows the generation of quantitative maps, which facilitate the assessment of myelination and brain development [4, 14, 17]. Using the MDME sequence, tissue-specific parameters, such as T1-relaxation constants, T2-relaxation constants, and PD of the brain, can be acquired in less than 6 min [13, 16, 18-24]. Contrary to conventional MRI techniques, repetition time (TR), echo time (TE), and inversion time are not predefined in this method, as these factors are definable and adjustable in retrospect [15, 20]. Subsequently, SyMRI generates the desired contrasts within a few seconds [13]. Thus, it would be conceivable to apply SyMRI in the clinical routine. Previously published data show that T1 and T2weighted contrasts generated by SyMRI are comparable to those of conventionally acquired MR image data [15, 19]. This technique has already been investigated as a diagnostic tool in a variety of conditions, primarily in neurological diseases [25-28]; however, the majority of studies referred exclusively to adults. Thus, further data on the applicability of SyMRI in children and neonates are of particular importance.

The aim of this study was to evaluate the clinical practicability of SyMRI-generated MR contrasts for the assessment of the neonatal brain. For this purpose, four neuroradiologists, independently rating, assessed a variety of cases based on T1 and T2-weighted images generated by SyMRI and conventional T1 and T2-weighted sequences. The sensitivity and specificity of conventional MRI and the MR data post-processing software SyMRI were calculated and compared in a neonatal cohort.

\section{Material and Methods}

\section{Ethical Approval}

The protocol of this study was approved by the local Ethics Commission and performed in accordance with the Declaration of Helsinki.

\section{Study Cohort}

We retrospectively collected 36 cases of neonatal MRI, which included T1 and T2-weighted sequences, as well as MDME sequences. The data acquisition was performed at the neuroradiology department of a tertiary care hospital between June 2017 and August 2019. All newborns were referred for MRI examination by the Department of Pediatrics and Adolescent Medicine, Division of Neonatology, Pediatric Intensive Care and Neuropediatrics. At this institution, every preterm infant is subjected to MRI at approximately term-equivalent age, regardless of clinical presentation or previous ultrasound findings. Term-born infants were examined postnatally between 2 days and 12 weeks postpartum. Indications for the MRI included, e.g., a diagnosis or suspicion of intraventricular hemorrhage, hypoxic ischemic encephalopathy, and cerebral infarction. By means of the electronic patient documentation system, the clinical information of the subjects was retrospectively obtained. After careful review, a total of 32 neonates, $50 \%$ of which were inconspicuous cases (16/32), were included in this study. Of 36 subjects, 4 subjects had to be excluded due to lack of clinical information and 2 of 16 inconspicuous subjects were term-born. Clinical follow-up examinations confirmed an unimpaired neurological development. Clinical characteristics and demographic information of the included neonates are shown in Table 1.

\section{Data Acquisition, MDME Sequence, and MR Post- processing}

In order to prevent movement artifacts, infants were fed 30 min prior to the MRI examination and bedded in a vac-

Table 1 Demographics and clinical characteristics

\begin{tabular}{ll}
\hline & $\begin{array}{l}\text { Neonates } \\
n=32\end{array}$ \\
\hline Characteristics/demography & \\
Male/female & $16 / 16$ \\
Term born/preterm & $8 / 24$ \\
Gestational age (weeks) & a \\
Clinical diagnosis & $27+5(23+3-41+5)$ \\
Inconspicuous $^{\mathrm{b}}$ & \\
Hemorrhage $^{\mathrm{b}, \mathrm{c}}$ & $n=16$ \\
Expired infarction $^{\mathrm{b}}$ & $n=9$ \\
HIE $^{\mathrm{b}}$ & $n=2$ \\
PVL $^{\mathrm{b}}$ & $n=3$ \\
Venous vessel malformation $^{\mathrm{b}}$ & $n=1$ \\
\hline
\end{tabular}

$H I E$ hypoxic-ischemic encephalopathy, $P V L$ periventricular leukomalacia

a Data represented as median and range

${ }^{\mathrm{b}}$ Data represented as total number

'Including hyperacute, acute, subacute, and chronic intraventricular, cortical, subcortical, parenchymal, and subdural hemorrhage 
Table 2 MRI sequences and technical features

\begin{tabular}{|c|c|c|c|c|c|c|c|}
\hline Sequence & Plane & Matrix (slices) & FOV (mm) & Voxel size $(\mathrm{mm})$ & $\mathrm{TR}(\mathrm{ms})$ & $\mathrm{TE}(\mathrm{ms})$ & AT \\
\hline T1 SE & Axial & $144 \times 115 \times 30$ & $120 \times 120 \times 90$ & $0.83 \times 1.05 \times 3$ & 400 & 15 & $3: 07$ \\
\hline T2 TSE & Axial & $128 \times 113 \times 34$ & $120 \times 120 \times 102$ & $0.94 \times 1.06 \times 3$ & 3000 & 140 & $1: 48$ \\
\hline T2 TSE & Coronal & $116 \times 103 \times 36$ & $110 \times 110 \times 108$ & $0.94 \times 1.06 \times 3$ & 3000 & 140 & $1: 48$ \\
\hline T2 TSE & Sagittal & $128 \times 113 \times 36$ & $120 \times 120 \times 108$ & $0.94 \times 1.06 \times 3$ & 3000 & 140 & $1: 48$ \\
\hline DWI & Axial & $176 \times 170 \times 28$ & $200 \times 200 \times 92$ & $1.14 \times 1.15 \times 3$ & 4066 & 90 & $1: 34$ \\
\hline SWI & Axial & $200 \times 138 \times 90$ & $170 \times 139 \times 90$ & $0.85 \times 1 \times 2$ & 51 & 12 & $3: 35$ \\
\hline $\mathrm{T} 1$ (3D) & Sagittal & $160 \times 160 \times 99$ & $120 \times 120 \times 99$ & $0.75 \times 0.75 \times 2$ & 25 & 7.6 & $3: 46$ \\
\hline MDME & Axial & $224 \times 159 \times 22$ & $200 \times 165 \times 109$ & $0.9 \times 1 \times 4$ & 3309 & 13 & $5: 24$ \\
\hline
\end{tabular}

$A T$ acquisition time, $D W I$ diffusion-weighted imaging, $F O V$ field-of-view, $M D M E$ multi-dynamic multi-echo sequence, $S E$ spin echo, $S W I$ susceptibility-weighted imaging, $T E$ echo time, TR repetition time, TSE turbo spin echo

uum mattress. In cases of routine MRI due to prematurity, no sedation was used. In cases of suspected or diagnosed brain pathologies, a sedation using chloral hydrate $(30-50 \mathrm{mg} / \mathrm{kg})$ or chloral hydrate combined with midazolam $(0.1 \mathrm{mg} / \mathrm{kg})$ was used. All included subjects were examined using a standardized neonatal MRI protocol (T1 spin echo [SE] sequence [single plane], T2 turbo spin echo [TSE] sequence [three orthogonal planes], diffusionweighted imaging [DWI] sequence, susceptibility-weighted imaging [SWI] sequence, T1 3D sequence) on a Philips Ingenia (Philips Healthcare, Best, The Netherlands) $1.5 \mathrm{~T}$ MR system. In addition, an MDME sequence (single plane) was acquired. The sequence determines T1 and T2-relaxation constants, as well as PD of the examined tissue, by applying two repeated acquisition phases [13, 29]. During the first phase, a slice-selective saturation pulse (flip angle: $120^{\circ}$ ) was used to saturate one slice. During the second phase, slice-selective excitation pulses (flip angle: $90^{\circ}$ ) and slice-selective refocusing pulses (flip angle: $180^{\circ}$ ) were used to generate a train of spin echoes for another slice $[13,16,29]$. Based on the mismatch between the saturated slice and the image slice, a matrix with various effects of T1- and T2-relaxation rates was acquired [13, 29]. Echo trains characterized by different saturation delays allowed the T1- and T2-relaxation parameters to be estimated [13, 16, 29]. The ascertained T1-relaxation constants also enabled the calculation of the local radiofrequency field (B1), which allowed the correction of flip angle deviations. Based on the acquired relaxation parameters and B1, the PD can be calculated [16]. The MDME sequence-based MR postprocessing was performed by applying SyMRI (Version 11.1.5) to generate $\mathrm{T} 1$-weighted (preset $\mathrm{TR}=650 \mathrm{~ms}$; preset $\mathrm{TE}=10 \mathrm{~ms}$ ) and $\mathrm{T} 2$-weighted (preset $\mathrm{TR}=4500 \mathrm{~ms}$; preset $\mathrm{TE}=100 \mathrm{~ms}$ ) contrasts. Table 2 gives an overview about the technical features of the individual sequences.

\section{Assessment of the Neonatal Brain}

In order to compare conventional MR contrasts and SyMRIgenerated MR contrasts (Figs. 1 and 2), the included sub- jects were divided into two groups. Each group $(n=16)$ included $50 \%$ inconspicuous cases (8/16) and 50\% pathological cases (8/16). Table 3 shows the allocation of pathological cases to the groups. For the assessment of image data, four independent investigators were consulted (rater 1: 15 years of experience in assessing neonatal MRI; rater 2: 6 years of experience in assessing neonatal MRI; raters 3 and 4: limited experience in assessing neonatal MRI). All raters were blinded to the diagnosis of the included neonates. Each group was evaluated by an experienced and a less experienced investigator on SyMRI-generated, as well as on conventionally acquired axial T1- and T2-weighted contrasts. Hence, raters 1 and 3 assessed the first group based on conventional T1- and T2-weighted images, whereas raters 2 and 4 assessed the same group based on SyMRI-generated T1- and T2-weighted contrasts. Conversely, raters 1 and 3 assessed the second group based on SyMRI-generated T1 and T2-weighted contrasts and raters 2 and 4 assessed the same group based on conventionally acquired T1- and T2-weighted images (Fig. 3). Based on the provided image data, raters had to make the correct diagnosis. In addition, the assessing neuroradiologists obtained information about the gestational age at birth of the subjects, as well as the corresponding indication for the MRI examination. The raters were asked about signs of hemorrhage, infarction, or hypoxic-ischemic encephalopathy, if the correct diagnosis was deducible based on the referral. Available follow-up MRI examinations and other imaging modalities allowed the clarification of the correct diagnosis in advance. During the assessment of conventional images, the evaluators had the opportunity to adjust the windowing at their discretion. In the case of SyMRIgenerated contrasts, the raters had the option to modulate TR and TE, in order to generate appropriate images.

\section{Statistical Analyses}

Statistical analyses were performed using XLSTAT 2017 Version 20.5 (Addinsoft, Paris, France) and SPSS Statistics for Macintosh Version 25.0 (IBM Corporation, Ar- 
Fig. 1 Inconspicuous case: brain of a former preterm infant $(24+4$ weeks). The left column $(\mathbf{a}, \mathbf{b})$ shows conventionally acquired T1-weighted (a) and T2-weighted contrasts (b). The right column (c, d) shows T1-weighted (c) and T2-weighted (d) contrasts generated by the MR data post-processing software SyMRI
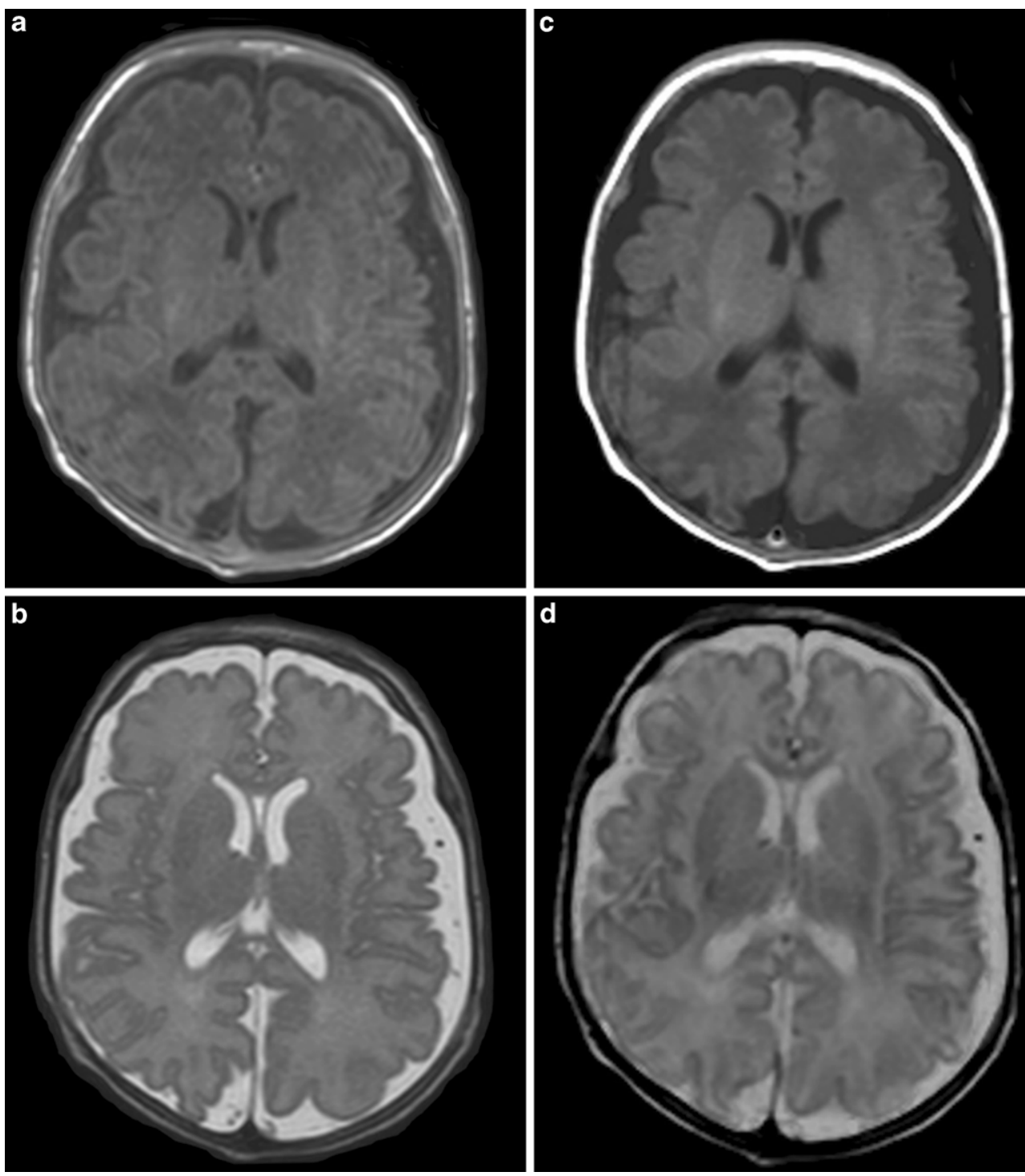

monk, NY, USA) at a significance level of alpha $(\alpha)=5 \%$ $(p<0.05)$. Graphs were created using XLSTAT 2017 Version 20.5.

The sensitivity and specificity for both conventionally acquired and SyMRI-generated T1 and T2-weighted contrasts were calculated separately based on the evaluation of experienced and less experienced raters. Furthermore, total values for sensitivity and specificity were calculated on the basis of the assessment of all raters.

An interrater reliability was calculated using the Cohen coefficient $(\mathrm{C} \kappa)$ and the Fleiss coefficient $(\mathrm{F} \kappa)$. The $\mathrm{C} \kappa$ was used to detect concordances between the assessment of raters 1 and 2 (experienced) and raters 3 and 4 (less experienced). The FK was used to detect the concordances of the assessment of all raters. According to Landis and Koch, $\kappa$ was interpreted as follows: $\kappa \leq 0$ : poor agreement; $0<\kappa \leq 0.2$ : slight agreement; $0.2<\kappa \leq 0.4$ : fair agreement; $0.4<\kappa \leq 0.6$ : moderate agreement; $0.6<\kappa \leq 0.8$ : substantial agreement; $0.8<\kappa \leq 1$ : (almost) perfect agreement [30].

The determined values were complemented by the corresponding $95 \%$ confidence interval (CI).

\section{Results}

\section{Diagnostic Accuracy, Sensitivity, and Specificity}

On both conventional and SyMRI-generated T1 and T2weighted images, $31 / 32$ cases $(96.9 \%)$ were correctly diagnosed or identified as inconspicuous by raters 1 and 2 (Table 4). With respect to the assessment of experienced raters, the sensitivity and specificity of conventional and SyMRI-generated MR contrasts did not show any differences (SyMRI: sensitivity: 0.94 [CI: 0.72-0.99]; speci- 

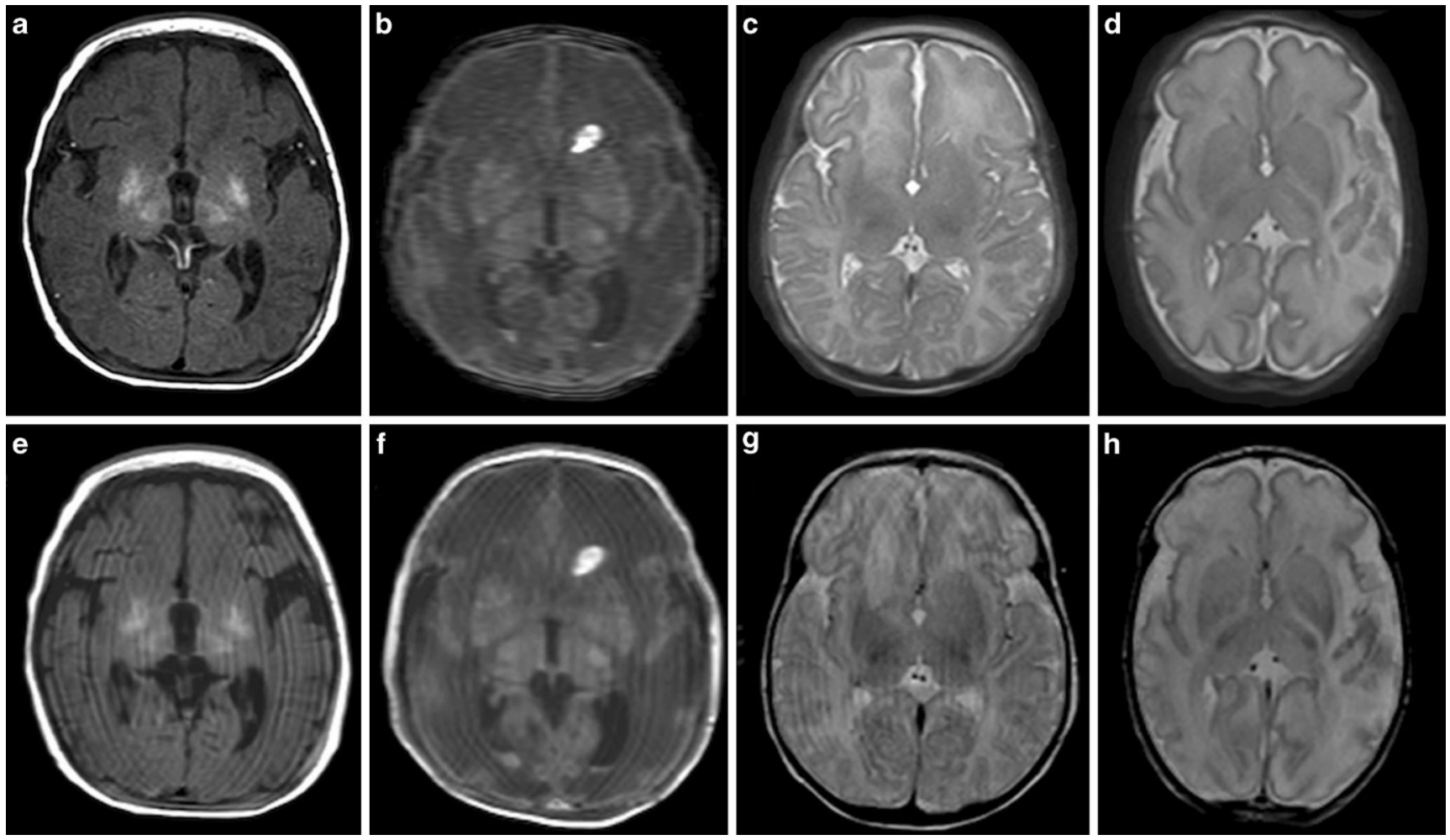

Fig. 2 Selection of pathological cases. The upper row shows conventional MR contrasts (a-d). The bottom row shows SyMRI-generated MR contrasts $(\mathbf{e}-\mathbf{h})$. a, e Hypoxic ischemic encephalopathy in a term-born infant $(39+4$ weeks) (T1-weighted contrast). b, f Former preterm neonate $(34+6$ weeks) with multifocal intracranial hemorrhage (T1-weighted contrast). c, $\mathbf{g}$ Term-born infant $(41+5$ weeks $)$ with expired infarction in the right caudate nucleus (T2-weighted contrast). d, h Former preterm neonate $(32+4$ weeks) with infarction of the left hemisphere (T2-weighted contrast)

ficity: 1 [CI $0.81-1]$ /conventional MRI: sensitivity: 0.94 [CI: 0.72-0.99]; specificity: 1 [CI: 0.81-1]; Fig. 4a).

On both conventional and SyMRI-generated T1 and T2weighted images, 29/32 (90.6\%) cases were correctly diagnosed or identified as inconspicuous by raters 3 and 4

Table 3 Group allocation

\begin{tabular}{llll}
\hline Group A $^{\mathrm{a}}$ & & & Group B \\
${$\cline { 1 - 2 }$} }$ & $n=16$ & $\begin{array}{l}\text { Condition/ } \\
\text { pathology }\end{array}$ & $n=16$ \\
\hline Inconspicuous $^{\mathrm{c}}$ & $n=8$ & Inconspicuous $^{\mathrm{c}}$ & $n=8$ \\
Hemorrhage $^{\mathrm{c}}$ & $n=5$ & Hemorrhage $^{\mathrm{c}}$ & $n=4$ \\
Expired infarction $^{\mathrm{c}}$ & $n=1$ & $\begin{array}{l}\text { Expired } \\
\text { infarction }^{\mathrm{c}}\end{array}$ & $n=1$ \\
HIE $^{\mathrm{c}}$ & $n=1$ & HIE $^{\mathrm{c}}$ & $n=2$ \\
Venous vessel $_{\text {malformation }^{\mathrm{c}}}$ & $n=1$ & PVL $^{\mathrm{c}}$ & $n=1$
\end{tabular}

HIE hypoxic ischemic encephalopathy, $P V L$ periventricular leukomalacia

${ }^{a}$ Assessed by raters 1 and 3 on the basis of conventionally acquired T1- and T2-weighted contrasts. Assessed by raters 2 and 4 based on SyMRI-generated T1- and T2-weighted contrasts

${ }^{\mathrm{b}}$ Assessed by raters 1 and 3 on the basis of SyMRI-generated T1- and T2-weighted contrasts. Assessed by raters 2 and 4 based on conventionally acquired $\mathrm{T} 1$ - and $\mathrm{T} 2$-weighted contrasts

${ }^{c}$ Data represented as total number
(Table 4). With respect to the assessment of less experienced raters, the sensitivity and specificity of conventional and SyMRI-generated MR contrasts differed (SyMRI: sensitivity: 0.81 [CI: 0.57-0.93]; specificity: 1 [CI 0.81-1]/ conventional MRI: sensitivity: 0.94 [CI: 0.72-0.99]; specificity: 0.88 [CI: 0.64-0.96]; Fig. 4b).

The total values (all raters) for sensitivity and specificity differed between SyMRI-generated and conventionally acquired contrasts (SyMRI: sensitivity: 0.88 [CI: 0.72-0.95]; specificity: 1 [CI $0.89-1]$ /conventional MRI: sensitivity: 0.94 [CI: 0.80-0.98]; specificity: 0.94 [CI: 0.80-0.98]; Fig. 4c).

\section{Interrater Statistics}

Based on the assessment of both conventionally acquired and SyMRI-generated T1-weighted and T2-weighted contrasts, there was a perfect agreement between the experienced raters, raters 1 and 2 (CK $=1$ [CI: 0.99-1], $p \leq 0.001$; [30]).

Based on the assessment of both conventionally acquired and SyMRI-generated T1-weighted and T2-weighted contrasts, there was a fair agreement between the less expe- 


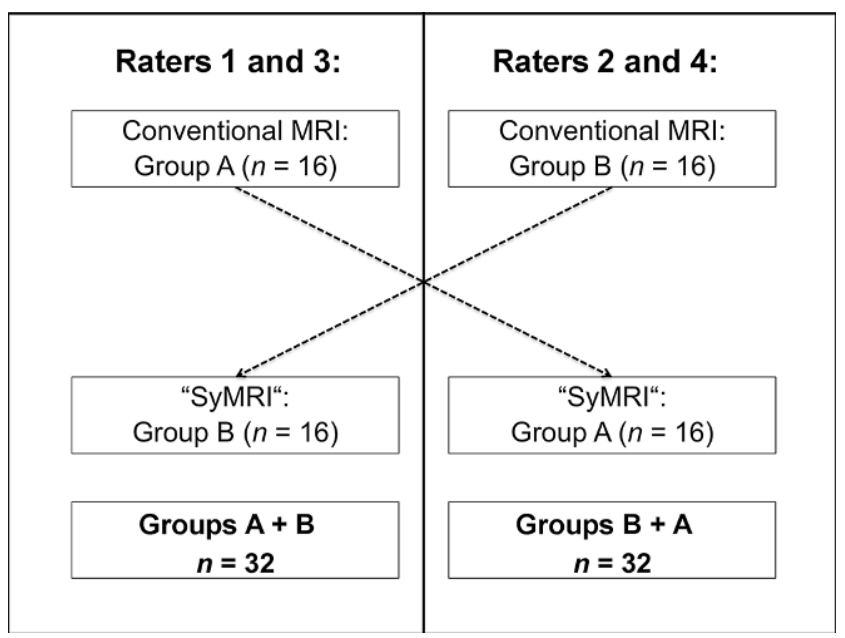

Fig. 3 Allocation of the groups to the corresponding raters

rienced raters, raters 3 and $4(\mathrm{C} \kappa=0.26$ [CI: 0.25-0.27], $p=0.135 ;[30])$.

On the basis of the assessment of both conventionally acquired and SyMRI-generated T1-weighted and T2weighted contrasts, there was a moderate agreement between all raters ( $\mathrm{F} \kappa=0.47$ [CI: $0.32-0.61], p \leq 0.001$; [30]).

\section{Discussion}

In this study the applicability of MR contrasts generated by the MR data post-processing software SyMRI was investigated in a clinical, radiological, diagnostic setting. Overall, SyMRI-generated T1-weighted and T2-weighted contrasts showed a lower sensitivity, but higher specificity, than conventional MR contrasts in a neonatal cohort (Fig. 4c). Interestingly, when sub-analyzing the evaluation by experienced raters, no differences between the two methods were found (Fig. 4a). In contrast, when evaluated by novice raters, conventionally acquired MR contrasts showed a higher sensitivity, whereas SyMRI-generated contrasts showed a higher

\section{a \\ Experienced}

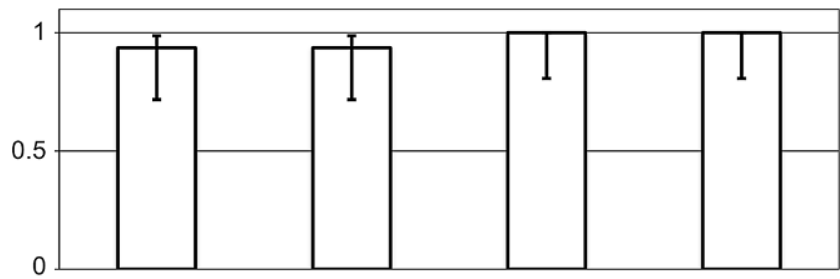

bovice
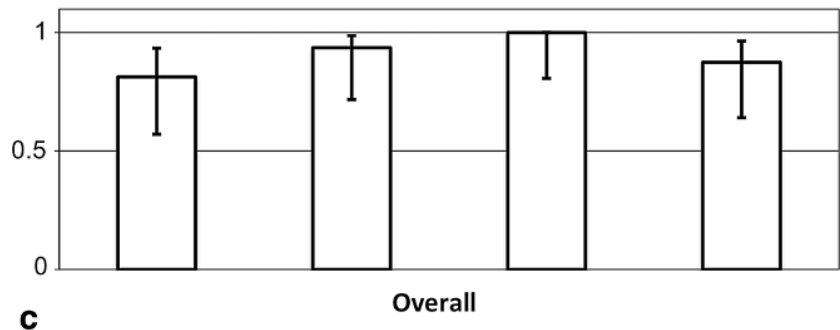

C

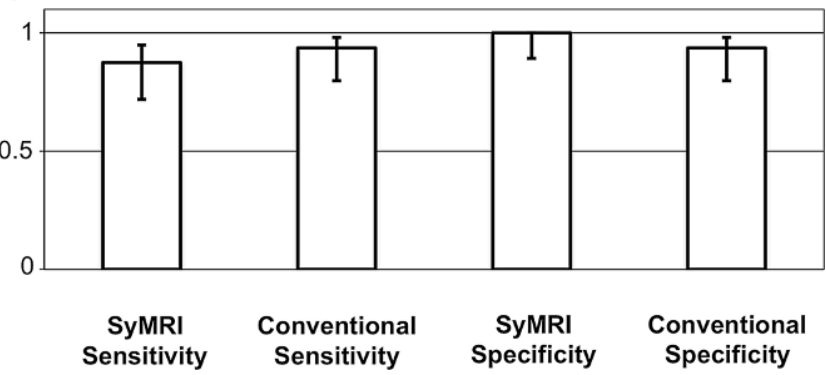

Fig. 4 a Sensitivity and specificity of conventional MRI and SyMRI on the basis of the assessment of experienced raters. b Sensitivity and specificity of conventional MRI and SyMRI on the basis of the assessment of novice raters. $\mathbf{c}$ Sensitivity and specificity of conventional MRI and SyMRI on the basis of the assessment of all raters

specificity (Fig. 4b). Based on the data presented here, the application of SyMRI in neonates appears to be comparable to conventional T1-weighted and T2-weighted sequences and suitable in a clinical setting. In addition, further MR contrasts and quantitative image data are provided for radiological diagnostic assessment, without extending examination time and post-processing time.

Table 4 Incorrectly identified cases

\begin{tabular}{lllll}
\hline Missed & Rater & Modality & Suspected & Group \\
\hline Inconspicuous & $3^{\mathrm{a}}$ & Conventional MRI & HIE & A \\
Expired infarction & $4^{\mathrm{a}}$ & SyMRI & Inconspicuous & Inconspicuous \\
PVL & $3^{\mathrm{a}}$ & SyMRI & HIE & B \\
Inconspicuous & $4^{\mathrm{a}}$ & Conventional MRI & Inconspicuous & B \\
Expired infarction & $1^{\mathrm{b}}$ & SyMRI & & B \\
& $2^{\mathrm{b}}$ & Conventional MRI & & \\
& $3^{\mathrm{a}}$ & SyMRI & & \\
& $4^{\mathrm{a}}$ & Conventional MRI & & \\
\hline
\end{tabular}

$H I E$ hypoxic ischemic encephalopathy, $P V L$ periventricular leukomalacia

${ }^{a}$ Novice rater

${ }^{\mathrm{b}}$ Experienced rater 
As described in the literature, various types of brain damage that can occur in the neonatal period may severely affect further neurodevelopment [2, 31-34]. There is evidence that hemorrhage, hypoxic ischemic encephalopathy, and infarction are primarily among the most common causes of neonatal morbidity and mortality [35, 36]. These conditions are highly associated with preterm birth [37]. In order to best reflect the clinical reality, preterm infants and frequently observed pathologies were included in this study. In addition to conventionally acquired MR contrasts, SyMRI was applied to provide T1 and T2-weighted images. The software generates the contrasts based on the ascertained relaxation parameters and $\mathrm{PD}$, determined by a single acquisition of an MDME sequence [16, 18].

These results are in line with several descriptions in the literature $[15,19]$. The clinical application and the diagnostic accuracy of the method have already been investigated in neurological disorders in adults. It could be shown that contrasts generated by SyMRI were not inferior to those of conventionally acquired contrasts [15]; however, there are still few data on the use of this software in children and neonates. West et al. applied this technique in a cohort of 32 patients with a mean age of 12.6 years [38]. Furthermore, there are descriptions of the use of SyMRI in a cohort of 29 patients with a median age of 6 years [39]. Both studies showed that the method provides acceptable and reliable results [38, 39]. Nevertheless, on the basis of the assessment of novices, sensitivity and specificity differed and a poor agreement between both novice raters was observed. This finding is in accordance with descriptions in the literature [40, 41]. Since a perfect concordance for the evaluation of experienced neuroradiologists was observed, the poor agreement of novice raters was attributed to the lack of experience in the assessment of the neonatal brain [30]. Thus, based on the varying level of experience, there was a moderate agreement for the assessment of all raters [30].

Generally, experienced raters made more use of the possibility to modulate TR and TE, whereas novices tended to evaluate the images on the basis of the default preset values. Particularly when assessing myelination, an adaption of the scan parameters seems sensible. A short TR/TE MR contrast shows the presence of myelinated structures as hyperintense areas and enables the process of myelination in the course of development to be tracked. In contrast, a long TR/TE image contrast is appropriate for assessing the quantity of myelin deposited [42]. According to descriptions in the literature, TR/TE of $3000 / 120 \mathrm{~ms}$ is best suited for determining the individual state of brain maturity in the first months after birth. Using this adjustment, myelinated white matter appears considerably hypointense compared to non-myelinated brain areas and gray matter [42]. Hence, an adjustment of TR/TE facilitates the assess- ment of brain maturity. Furthermore, experienced raters preferred short TR/TE contrasts in case of suspected hypoxic ischemic encephalopathy, which is in line with descriptions by Barkovich et al. [43]. As shown in Table 4, novices misdiagnosed inconspicuous cases, as they suspected mild hypoxic ischemic encephalopathy. An adjustment of TR/TE might have enabled a more precise assessment. Based on the data presented here, no recommendations on the use of technical parameters for assessing the neonatal brain can be derived; however, SyMRI represents a method that enables the investigation of beneficial technical adjustments for the diagnosis of cerebral pathologies and the visualization of myelination in neonates.

Primarily in adults, the quality of SyMRI-generated images appears to be equal to that of conventional MRI sequences [15]. Also, in a pediatric cohort, there is evidence that the quality of the images was not inferior to conventionally acquired MR contrasts [38]. Interestingly, Andica et al. reported that fluid-attenuated inversion recovery (FLAIR) contrasts generated by SyMRI were even superior in the diagnosis of neonatal meningitis compared to conventional FLAIR sequences [44]. Our data show that MDME sequence-based MR post-processing allows diagnostic accuracy but in most cases the image quality was inferior to that of conventional MR images (Fig. 2). A possible explanation for this fact is that MR contrasts generated by SyMRI are based on a single MDME sequence. Hence, movement during the data acquisition presents as motion artifact in all MDME-based images; however, optimal conditions allow the generation of high-quality SyMRI image data in a neonatal cohort, as shown in Fig. 1.

This software offers advantages that may be of interest, especially when assessing the neonatal brain. Compared to conventional MR image data, quantitative MR mapping allows a better assessment of myelination and brain maturity $[13,17,38]$. Based on the relaxation constants of the examined tissue, voxels are assigned to gray and white matter, as well as to cerebrospinal fluid [13]; however, using conventional MR techniques, the generation of quantitative MR maps is a highly time-consuming process and, therefore, not applicable in the clinical routine. The software provides quantitative maps in a clinically acceptable time, which offers further prospects in medical imaging [17]; however, SyMRI-based quantitative MR mapping was beyond the scope of the present study and should be the subject of future investigations.

Nonetheless, an important weakness of this method must be pointed out. As shown in Table 4, based on conventional MR contrasts, the identification of subtle infarctions (Fig. 2c, g) is highly challenging even for experts in the field of neuropediatric radiology. The SyMRI does not provide DWI for the reliable diagnosis of stroke; however, further studies are needed to investigate whether SyMRI may pro- 
vide information that proves beneficial for the detection of subtle cerebrovascular damage.

This study has several limitations. The technical properties of conventionally acquired and SyMRI-generated contrasts differed, especially regarding slice thickness and spatial resolution (Table 2). Hence, a direct comparison of both methods was limited to a certain extent. Due to the retrospective nature of the study, the sample size was relatively small. Furthermore, we included a high number of patients with hemorrhages, a condition that can be easily diagnosed, even by novices in the field of neonatal neuroradiology; however, it should be emphasized that hemorrhages represent one of the most common pathologies in preterm neonates [45].

In summary, our data indicate that SyMRI-generated T1weighted and T2-weighted contrasts serve as a reliable tool for the detection of neonatal brain pathologies. The method demonstrated diagnostic non-inferiority compared to the current clinical gold standard. We conclude that the MR data post-processing software SyMRI is clinically applicable for the assessment of the neonatal brain, while leading to a more efficient use of available imaging time.

Funding Open access funding provided by Medical University of Vienna.

Conflict of interest V. Schmidbauer, G. Geisl, M. Cardoso Diogo, S. Jengojan, V. Perepelov, M. Weber, K. Goeral, F. Lindenlaub, K. Klebermass-Schrehof, A. Berger, D. Prayer and G. Kasprian declare that they have no competing interests.

Open Access This article is licensed under a Creative Commons Attribution 4.0 International License, which permits use, sharing, adaptation, distribution and reproduction in any medium or format, as long as you give appropriate credit to the original author(s) and the source, provide a link to the Creative Commons licence, and indicate if changes were made. The images or other third party material in this article are included in the article's Creative Commons licence, unless indicated otherwise in a credit line to the material. If material is not included in the article's Creative Commons licence and your intended use is not permitted by statutory regulation or exceeds the permitted use, you will need to obtain permission directly from the copyright holder. To view a copy of this licence, visit http://creativecommons.org/licenses/by/4. $0 /$.

\section{References}

1. Saunders DE, Thompson C, Gunny R, Jones R, Cox T, Chong WK. Magnetic resonance imaging protocols for paediatric neuroradiology. Pediatr Radiol. 2007;37:789-97.

2. Dorner RA, Burton VJ, Allen MC, Robinson S, Soares BP. Preterm neuroimaging and neurodevelopmental outcome: a focus on intraventricular hemorrhage, post-hemorrhagic hydrocephalus, and associated brain injury. J Perinatol. 2018;38:1431-43.

3. Barkovich AJ, Kjos BO, Jackson DE, Norman D. Normal maturation of the neonatal and infant brain: MR imaging at $1.5 \mathrm{~T}$. Radiology. 1988;166:173-80.
4. Deoni SC, Mercure E, Blasi A, Gasston D, Thomson A, Johnson M, Williams SC, Murphy DG. Mapping infant brain myelination with magnetic resonance imaging. J Neurosci. 2011;31:784-91.

5. Doria V, Arichi T, Edwards DA. Magnetic resonance imaging of the preterm infant brain. Curr Pediatr Rev. 2014;10:48-55.

6. Laptook AR. Birth asphyxia and hypoxic-ischemic brain injury in the preterm infant. Clin Perinatol. 2016;43:529-45.

7. Ibrahim J, Mir I, Chalak L. Brain imaging in preterm infants $<32$ weeks gestation: a clinical review and algorithm for the use of cranial ultrasound and qualitative brain MRI. Pediatr Res. 2018;84:799-806.

8. Mathur A, Inder T. Magnetic resonance imaging-insights into brain injury and outcomes in premature infants. J Commun Disord. 2009;42:248-55.

9. Parikh NA. Advanced neuroimaging and its role in predicting neurodevelopmental outcomes in very preterm infants. Semin Perinatol. 2016;40:530-41.

10. Kidokoro H, Anderson PJ, Doyle LW, Woodward LJ, Neil JJ, Inder TE. Brain injury and altered brain growth in preterm infants: predictors and prognosis. Pediatrics. 2014;134:e444-53.

11. Rutherford M, Pennock J, Schwieso J, Cowan F, Dubowitz L. Hypoxic-ischaemic encephalopathy: early and late magnetic resonance imaging findings in relation to outcome. Arch Dis Child Fetal Neonatal Ed. 1996;75:F145-51.

12. Dyet LE, Kennea N, Counsell SJ, Maalouf EF, Ajayi-Obe M, Duggan PJ, Harrison M, Allsop JM, Hajnal J, Herlihy AH, Edwards B, Laroche S, Cowan FM, Rutherford MA, Edwards AD. Natural history of brain lesions in extremely preterm infants studied with serial magnetic resonance imaging from birth and neurodevelopmental assessment. Pediatrics. 2006;118:536-48.

13. Hagiwara A, Warntjes M, Hori M, Andica C, Nakazawa M, Kumamaru KK, Abe O, Aoki S. SyMRI of the brain: rapid quantification of relaxation rates and proton density, with Synthetic MRI, automatic brain segmentation, and myelin measurement. Invest Radiol. 2017;52:647-57.

14. McAllister A, Leach J, West H, Jones B, Zhang B, Serai S. Quantitative synthetic MRI in children: normative intracranial tissue segmentation values during development. AJNR Am J Neuroradiol. 2017;38:2364-72.

15. Tanenbaum LN, Tsiouris AJ, Johnson AN, Naidich TP, DeLano MC, Melhem ER, Quarterman P, Parameswaran SX, Shankaranarayanan A, Goyen M, Field AS. Synthetic MRI for clinical neuroimaging: results of the magnetic resonance image compilation (MAGiC) prospective, multicenter, multireader trial. AJNR Am J Neuroradiol. 2017;38:1103-10.

16. Warntjes JB, Leinhard OD, West J, Lundberg P. Rapid magnetic resonance quantification on the brain: optimization for clinical usage. Magn Reson Med. 2008;60:320-9.

17. Schmidbauer V, Geisl G, Diogo M, Weber M, Goeral K, Klebermass-Schrehof K, Berger A, Prayer D, Kasprian G. SyMRI detects delayed myelination in preterm neonates. Eur Radiol. 2019;29:7063-72.

18. Whittall KP, MacKay AL, Graeb DA, Nugent RA, Li DK, Paty DW. In vivo measurement of $\mathrm{T} 2$ distributions and water contents in normal human brain. Magn Reson Med. 1997;37:34-43.

19. Bobman SA, Riederer SJ, Lee JN, Suddarth SA, Wang HZ, MacFall JR. Synthesized MR images: comparison with acquired images. Radiology. 1985;155:731-8.

20. Bobman SA, Riederer SJ, Lee JN, Suddarth SA, Wang HZ, Drayer BP, MacFall JR. Cerebral magnetic resonance image synthesis. AJNR Am J Neuroradiol. 1985;6:265-9.

21. Riederer SJ, Suddarth SA, Bobman SA, Lee JN, Wang HZ, MacFall JR. Automated MR image synthesis: feasibility studies. Radiology. 1984;153:203-6.

22. Deichmann R. Fast high-resolution T1 mapping of the human brain. Magn Reson Med. 2005;54:20-7. 
23. Henderson E, McKinnon G, Lee TY, Rutt BK. A fast 3D looklocker method for volumetric T1 mapping. Magn Reson Imaging. 1999; 17:1163-71.

24. Neeb H, Zilles K, Shah NJ. A new method for fast quantitative mapping of absolute water content in vivo. Neuroimage. 2006;31:1156-68.

25. Wallaert L, Hagiwara A, Andica C, Hori M, Yamashiro K, Koshino S, Maekawa T, Kamagata K, Aoki S. The advantage of Synthetic MRI for the visualization of anterior temporal pole lesions on double inversion recovery (DIR), phase-sensitive inversion recovery (PSIR), and myelin images in a patient with CADASIL. Magn Reson Med Sci. 2018; 17:275-6.

26. Arita Y, Takahara T, Yoshida S, Kwee TC, Yajima S, Ishii C, Ishii R, Okuda S, Jinzaki M, Fujii Y. Quantitative assessment of bone metastasis in prostate cancer using synthetic magnetic resonance imaging. Invest Radiol. 2019;54:638-44.

27. Park M, Moon Y, Han SH, Kim HK, Moon WJ. Myelin loss in white matter hyperintensities and normal-appearing white matter of cognitively impaired patients: a quantitative synthetic magnetic resonance imaging study. Eur Radiol. 2019;29:4914-21.

28. Krauss W, Gunnarsson M, Nilsson M, Thunberg P. Conventional and synthetic MRI in multiple sclerosis: a comparative study. Eur Radiol. 2018;28:1692-700.

29. Kang KM, Choi SH, Kim H, Hwang M, Yo RE, Yun TJ, Kim JH, Sohn $\mathrm{CH}$. The effect of varying slice thickness and interslice gap on $\mathrm{T} 1$ and T2 measured with the multidynamic multiecho sequence. Magn Reson Med Sci. 2019;18:126-33.

30. Landis JR, Koch GG. The measurement of observer agreement for categorical data. Biometrics. 1977;33:159-74.

31. Bonifacio SL, Glass HC, Vanderpluym J, Agrawal AT, Xu D, Barkovich AJ, Ferriero DM. Perinatal events and early magnetic resonance imaging in therapeutic hypothermia. J Pediatr. 2011;158:360-5.

32. Jacobs SE, Berg M, Hunt R, Tarnow-Mordi WO, Inder TE, Davis PG. Cooling for newborns with hypoxic ischaemic encephalopathy. Cochrane Database Syst Rev. 2013 Jan 31;(1): CD003311.

33. Gilard V, Chadie A, Ferracci FX, Brasseur-Daudruy M, Proust F, Marret S, Curey S. Post hemorrhagic hydrocephalus and neurodevelopmental outcomes in a context of neonatal intraventricular hemorrhage: an institutional experience in 122 preterm children. BMC Pediatr. 2018;18:288.

34. Ecury-Goossen GM, van der Haer M, Smit LS, Feijen-Roon M, Lequin M, de Jonge RC, Govaert P, Dudink J. Neurodevelopmental outcome after neonatal perforator stroke. Dev Med Child Neurol. 2016;58:49-56.
35. Liu S, An N, Yang H, Yang M, Hou Z, Liu L, Liu Y. Pediatric intractable epilepsy syndromes: reason for early surgical intervention. Brain Dev. 2007;29:69-78.

36. Gale C, Statnikov Y, Jawad S, Uthaya SN, Modi N; Brain Injuries expert working group. Neonatal brain injuries in England: population-based incidence derived from routinely recorded clinical data held in the National Neonatal Research Database. Arch Dis Child Fetal Neonatal Ed. 2018;103:F301-6.

37. Hinojosa-Rodríguez M, Harmony T, Carrillo-Prado C, Van Horn JD, Irimia A, Torgerson C, Jacokes Z. Clinical neuroimaging in the preterm infant: diagnosis and prognosis. Neuroimage Clin. 2017;16:355-68.

38. West H, Leach JL, Jones BV, Care M, Radhakrishnan R, Merrow AC, Alvarado E, Serai SD. Clinical validation of synthetic brain MRI in children: initial experience. Neuroradiology. 2017;59:43-50.

39. Lee SM, Choi YH, Cheon JE, Kim IO, Cho SH, Kim WH, Kim HJ, Cho HH, You SK, Park SH, Hwang MJ. Image quality at synthetic brain magnetic resonance imaging in children. Pediatr Radiol. 2017;47:1638-47.

40. Fortin M, Dobrescu O, Jarzem P, Ouellet J, Weber MH. Quantitative magnetic resonance imaging analysis of the cervical spine extensor muscles: intrarater and interrater reliability of a novice and an experienced rater. Asian Spine J. 2018;12:94-102.

41. Prasad BP, Bhatta RC, Chaudhary J, Sharma S, Mishra S, Cuddapah PA, Stoller NE, Yu SN, Rahman SA, Deiner M, Keenan JD, Gaynor $\mathrm{BD}$. Agreement between novice and experienced trachoma graders improves after a single day of didactic training. Br J Ophthalmol. 2016;100:762-5.

42. van der Knaap MS, Valk J. Magnetic resonance of myelination and myelin disorders. 3rd ed. Berlin, Heidelberg, New York: Springer; 2005.

43. Barkovich AJ, Hajnal BL, Vigneron D, Sola A, Partridge JC, Allen F, Ferriero DM. Prediction of neuromotor outcome in perinatal asphyxia: evaluation of MR scoring systems. AJNR Am J Neuroradiol. 1998;19:143-9.

44. Andica C, Hagiwara A, Nakazawa M, Kumamaru KK, Hori M, Ikeno M, Shimizu T, Aoki S. Synthetic MR imaging in the diagnosis of bacterial meningitis. Magn Reson Med Sci. 2017;16:91-2.

45. Payne AH, Hintz SR, Hibbs AM, Walsh MC, Vohr BR, Bann CM, Wilson-Costello DE; Eunice Kennedy Shriver National Institute of Child Health and Human Development Neonatal Research Network. Neurodevelopmental outcomes of extremely low-gestational-age neonates with low-grade periventricular-intraventricular hemorrhage. JAMA Pediatr. 2013;167:451-9. 